\title{
Transitional Cell Carcinoma and Pseudocirrhosis- A Case Report and Review of the Literature
}

Gilad Borisovsky ${ }^{1} \quad$ Daniel A. Goldstein ${ }^{2} \quad$ Noa Eliakim-Raz ${ }^{3} \quad$ Shlomit R. Tamir ${ }^{1}$

Address for correspondence Gilad Borisovsky, MD, 9 Avtalyon Street Entrance Bet Apt. 2, Ramat Gan 52424, Israel (e-mail: Gil.borisovsky@gmail.com).
Abstract
Keywords
- pseudocirrhosis
- liver
- metastasis
- transitional cell
carcinoma
- chemotherapy
- complications
- portal vein
thrombosis.

Pseudocirrhosis is the occurrence of capsular retraction in patients with metastatic liver disease, usually of breast origin. It usually appears in these patients following chemotherapy and may result from shrinkage of the tumor, with scarring and nodular regeneration. In this article, we describe the first case of pseudocirrhosis, following treatment of transitional cell carcinoma metastatic to the liver. Portal vein thrombosis, a rare complication in pseudocirrhosis, was also detected in this patient.

\section{Case Presentation}

We present a case of a 72-year-old patient with metastatic transitional cell carcinoma (TCC). His medical history was significant for ischemic heart disease, with a previous myocardial infarction, benign prostatic hyperplasia and past smoking. A noncontrast CT scan obtained at a different hospital, following a car accident in July 2018, detected a mass between the stomach and the liver and diffuse retroperitoneal and mesenteric lymphadenopathy, with no obvious primary tumor. Subsequent biopsy from the abdominal mass and a para-aortic lymph node revealed urothelial carcinoma. A subsequent PET/CT scan (- Fig. 1A, B; G.E. healthcare PET/CT scanner, Discovery 710, year of production- 2016; neck-chest-abdomen-pelvis $\mathrm{CT}$ with intravenous injection of ultravist $370,85 \mathrm{~mL}$, and PET acquisition using F-18 FDG with

DOI https://doi.org/

$10.1055 / \mathrm{s}-0040-1713829$

ISSN 2581-9933. activity of $8 \mathrm{mCi}$; slice thickness of $2.5 \mathrm{~mm}$; radiation dose$11.55 \mathrm{mGy}$ ) found numerous metastatic lesions in the liver and bones in addition to the aforementioned widespread lymphadenopathy. The primary tumor could not be localized in the PET/CT scan. Bone marrow biopsy was consistent with chronic lymphocytic leukemia.

Following six cycles of gemcitabine and cisplatin chemotherapy, which were initiated in July 2018, a gradual decrease in size and attenuation of the liver metastases was seen on CT to their complete resolution in March 2019 ( - Fig. 2A, B; Philips Medical Brilliance ICT Scanner, year of production2015; noncontrast upper abdomen scan and postcontrast scan of the chest, abdomen and pelvis with IV injection of ultravist 370, $85 \mathrm{~mL}$; slice thickness of $2 \mathrm{~mm}$; radiation dose $18 / 1 \mathrm{mGy})$. The response in the liver and lymph nodes was followed by disease progression with new brain metastases.

(c) 2020. Indian Society of Gastrointestinal and Abdominal Radiology. This is an open access article published by Thieme under the terms of the Creative Commons Attribution-NonDerivative-NonCommercial-License, permitting copying and reproduction so long as the original work is given appropriate credit. Contents may not be used for commercial purposes, or adapted, remixed, transformed or built upon. (https://creativecommons.org/licenses/by-nc-nd/4.0/)

Thieme Medical and Scientific Publishers Pvt. Ltd., A-12, 2nd Floor, Sector 2, Noida-201301 UP, India 

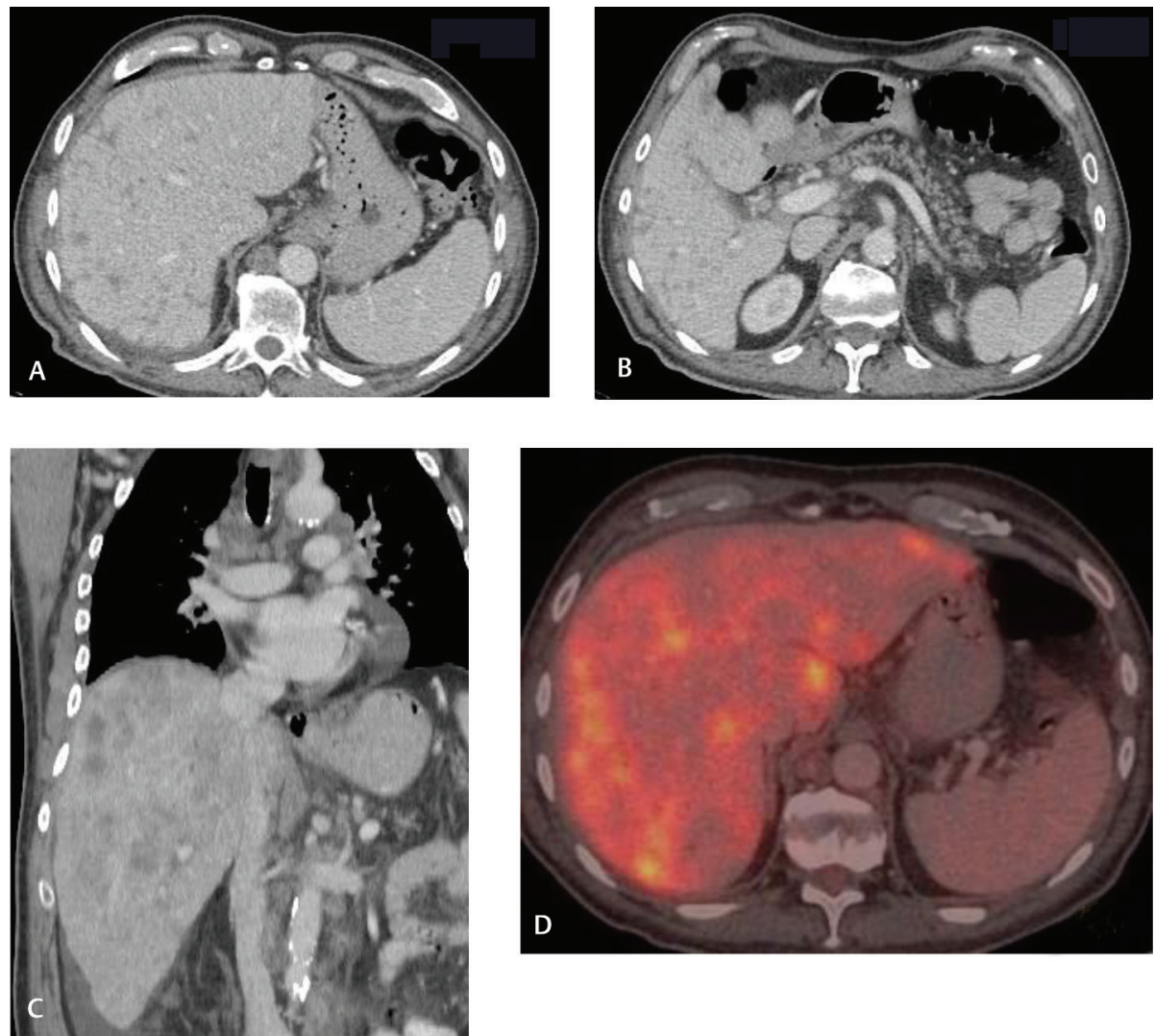

Fig. 1 (A, B) Axial images of portal venous phase CT scan of the abdomen, demonstrating hepatic metastases. (C, D) Coronal view and axial view: First PET-CT scan in the venous phase, demonstrating multiple FDG-avid hepatic metastases. FDG, fluorodeoxyglucose.
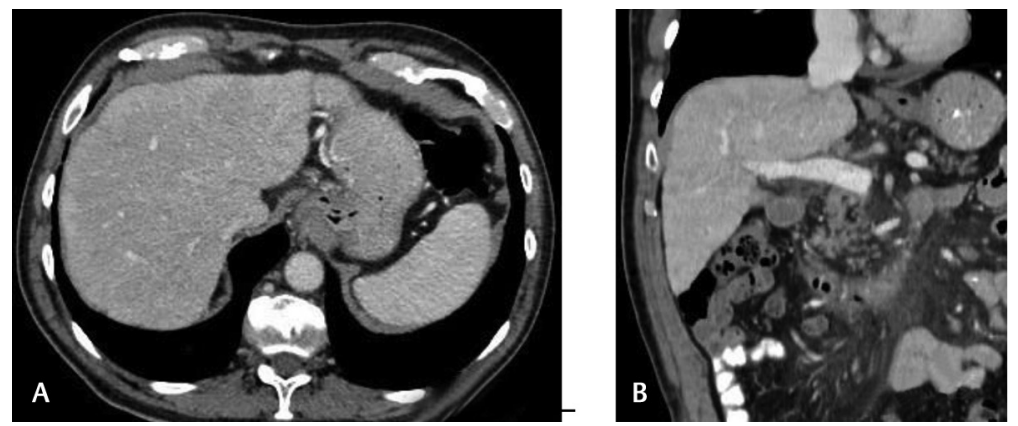

Fig. 2 (A, B) First CT scan in the venous phase after six cycles of chemotherapy demonstrate near resolution of the hepatic metastases (axial and coronal views).

In July 2019, the patient presented to the emergency department complaining of abdominal pain. An elevation of liver enzymes prompted an ultrasound scan which demonstrated ascites and a small liver with lobular borders and a nonuniform texture. The portal vein was measured $15 \mathrm{~mm}$ with a very slow flow of $12 \mathrm{~cm} /$ second. No portal thrombus was detected. The hepatic veins demonstrated a monophasic flow.

Further evaluation with a quadriphase CT scan ( - Fig. 3 A-E; G.E. healthcare CT scanner, Lightspeed VCT, year of production- 2006; upper abdomen before and after IV injection of ultravist $370,85 \mathrm{~mL}$, in the arterial phase followed by a scan of the chest, abdomen and pelvis in the venous phase and a delayed scan of the upper abdomen; slice thickness of $2.5 \mathrm{~mm}$ for the arterial and venous phases and $3.75 \mathrm{~mm}$ for the noncontrast and delayed phases; radiation dose- $79.91 \mathrm{mGy}$ ) showed a heterogeneous and nodular liver parenchyma with lobular contours. The portal vein diameter measured $18 \mathrm{~mm}$, thereby demonstrating portal venous hypertension. Ascites in moderate quantity were also noted. Considering the previous metastases to the liver, these findings are consistent with pseudocirrhosis. A new hypodense filling defect in the right main portal vein and its tributaries, with near complete obstruction. The filling defect did not demonstrate postcontrast enhancement, supporting a bland thrombus. A repeat ultrasound scan (- Fig. 3F; Siemens, ACUSON S2000, version VC25, year of production- 2012) shortly thereafter demonstrated a portal vein thrombus with no color flow on Doppler scan. This again supports a bland tumor. No biopsy was performed to the liver. It is worth mentioning that 

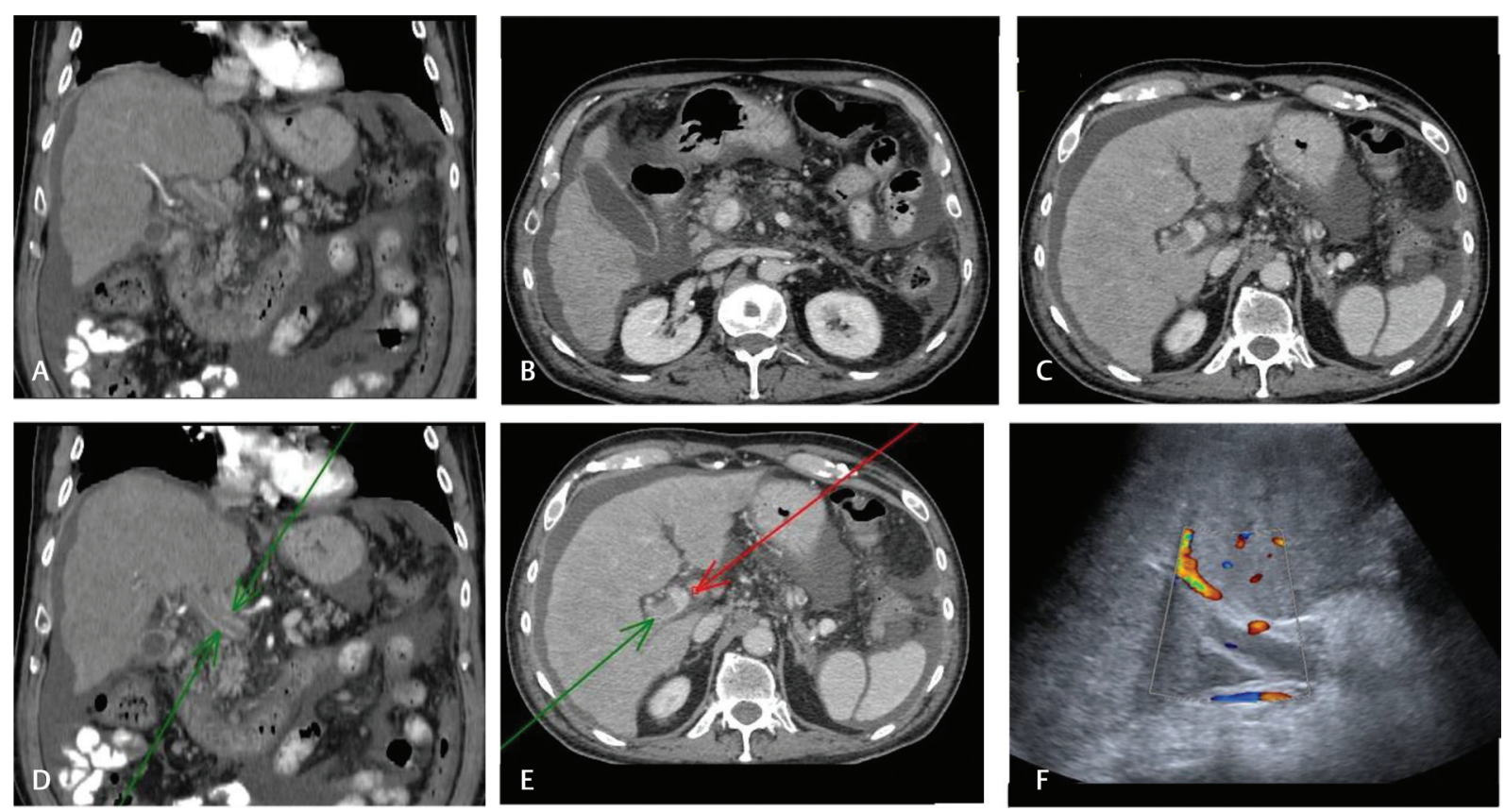

Fig. 3 (A-F) CT scan in the venous phase, axial and coronal views; (F) Doppler US scan. (A-C) Nodular liver with lobular margins and ascites. (D, E) Portal venous thrombosis. (F) Portal venous thrombosis without color flow on US scan.

the ascitic fluid was sampled and revealed malignant epithelial cells.

The patient passed away approximately 10 days following the quadriphase CT scan.

\section{Discussion}

Pseudocirrhosis describes the development of diffuse hepatic nodularity and segmental volume loss, following chemotherapeutic treatment for hepatic metastases. ${ }^{1,2}$ These changes cause capsular retraction and enlargement of the caudate lobe in a similar manner to cirrhosis. ${ }^{3}$ Even though pseudocirrhosis may induce portal hypertension and liver failure, it does not share the feature of loss of synthetic function with proper cirrhosis. ${ }^{3}$ Capsular retraction may evolve rapidly over 1 to 3 months. ${ }^{4}$

The underlying mechanisms for this phenomenon have not yet been adequately elucidated, although it is believed to result from shrinkage of tumor with scarring and nodular regeneration of uninvolved liver parenchyma. ${ }^{4}$ Fennesy et al found that pseudocirrhosis occurred more frequently in patients with larger metastases. They also demonstrated that capsular retraction occurs with an increase in the dimensions of a subjacent metastasis, possibly owing to local distortion of the liver parenchyma by fibrosis and tumor infiltration. ${ }^{5}$

Interestingly, pseudocirrhosis has been found mainly in patients with metastatic breast carcinoma. ${ }^{4,6}$ Pseudocirrhosis has also been sporadically reported in patients with other metastatic malignancies such as cancers of the digestive system (esophageal, stomach colon, pancreas,), thyroid and small-cell lung. ${ }^{7-12}$ Certain lymphoma types may masquerade as pseudocirrhosis secondary to desmoplastic reaction to tumor cells infiltrating the liver parenchyma. ${ }^{13}$
Portal vein thrombosis occurs in up to $26 \%$ of patients with cirrhosis, more commonly among those with more severe liver disease. ${ }^{14,15}$ In a review of the literature, we found only one case of portal vein thrombosis in pseudocirrhosis due to recurrent breast cancer. ${ }^{16}$

The differential diagnosis in our patient includes pseudocirrhosis, true cirrhosis, nodular regenerative hyperplasia, and drug-induced liver disease. Cirrhosis results from disruption of the hepatic architecture secondary to regenerating nodules and fibrosis, leading to a nodular liver contour and capsular retraction. ${ }^{17}$ In nodular regenerative hyperplasia (NRH), small regenerative nodules develop in the liver with scarce fibrosis secondary to altered blood flow. ${ }^{18}$ The etiology is variable, ranging from systemic conditions to drugs such as chemotherapeutic agents (most commonly azathioprine). Symptomatic portal hypertension is not very common in NRH. Imaging of drug-induced liver injury is often nonspecific with heterogeneous enhancement of the liver parenchyma, periportal edema and ascites. ${ }^{19}$

The constellation of nodular liver with capsular retraction and portal hypertension, with its complications in a patient with previous metastatic liver disease, treated with chemotherapy strongly favors the working diagnosis of pseudocirrhosis. We suggest this as the first known occurrence of pseudocirrhosis, following treatment of transitional cell carcinoma metastatic to the liver.

In addition, to our knowledge, portal vein thrombosis has not been described in patients with pseudocirrhosis. In our view, it resulted in a similar manner as in true cirrhosis; hence, the slow blood flow in the portal vein in combination with a hypercoagulable state predisposed to its occurrence in our patient. 


\section{Learning Points}

- Pseudocirrhosis is the occurrence of capsular retraction in patients with metastatic liver disease, accompanied by portal venous hypertension and ascites, usually with preserved synthetic function.

- Pseudocirrhosis occurs mainly in patients with metastatic breast carcinoma and, rarely, in hepatic metastases from other origins.

- Pseudocirrhosis occurring following treatment of TCC metastases in the liver is not commonly described.

- Portal venous thrombosis is a rare but important complication of pseudocirrhosis secondary to hepatic metastases.

\section{Conflict of Interest}

None declared.

\section{References}

1 Jeong WK, Choi SY, Kim J. Pseudocirrhosis as a complication after chemotherapy for hepatic metastasis from breast cancer. Clin Mol Hepatol 2013;19(2):190-194

2 Lipson JA, Qayyum A, Avrin DE, Westphalen A, Yeh BM, Coakley FVCT. CT and MRI of hepatic contour abnormalities. AJR Am J Roentgenol 2005;184(1):75-81

3 Jha P, Poder L, Wang ZJ, Westphalen AC, Yeh BM, Coakley FV. Radiologic mimics of cirrhosis. AJR Am J Roentgenol 2010;194(4):993-999

4 Young ST, Paulson EK, Washington K, Gulliver DJ, Vredenburgh $\mathrm{JJ}$, Baker ME. CT of the liver in patients with metastatic breast carcinoma treated by chemotherapy: findings simulating cirrhosis. AJR Am J Roentgenol 1994;163(6):1385-1388

5 Fennessy FM, Mortele KJ, Kluckert T, et al. Hepatic capsular retraction in metastatic carcinoma of the breast occurring with increase or decrease in size of subjacent metastasis. AJR Am J Roentgenol 2004;182(3):651-655

6 Qayyum A, Lee GK, Yeh BM, Allen JN, Venook AP, Coakley FV. Frequency of hepatic contour abnormalities and signs of portal hypertension at $\mathrm{CT}$ in patients receiving chemotherapy for breast cancer metastatic to the liver. Clin Imaging 2007;31(1):6-10
7 Kang SP, Taddei T, McLennan B, Lacy J. Pseudocirrhosis in a pancreatic cancer patient with liver metastases: a case report of complete resolution of pseudocirrhosis with an early recognition and management. World J Gastroenterol 2008;14(10):1622-1624

8 Mitani S, Kadowki S, Taniquchi H, Muto H, Muro K. Pseudocirrhosis in gastric cancer with diffuse liver metastases after a dramatic response to chemotherapy. Case Rep Oncol 2016;9(1):106-111

9 Kobashigawa C, Nakamoto M, Hokama A, Hirata T, Kinjo F, Fujita J. Pseudocirrhosis in metastatic esophageal cancer. South Med J 2010;103(5):488-489

10 Battisti S, Guida FM, Pagliara E, Tonini G, Zobel BB, Santini D. Pseudocirrhosis after anti-EGFR-based neoadjuvant therapy for hepatic metastasis from colon cancer: a different point of view. Clin Colorectal Cancer 2014;13(3):e13-e15

11 Ojeda VJ. Metastatic oat cell carcinoma simulating liver cirrhosis. N Z Med J 1977;86(600):480-481

12 Harry BL, Smith ML, Burton JR, Dasari A, Eckhardt SG, Diamond JR. Medullary thyroid cancer and pseudocirrhosis: case report and literature review. Curr Oncol 2012;19(1):e36-e41

13 Cohen J, Hariton E, Kothari D, Pihan GA, Robson SC. Hepatosplenic alpha/beta T-cell lymphoma masquerading as cirrhosis. J Gastrointest Oncol 2013;4(2):131-136

14 Amitrano L, Guardascione MA, Brancaccio V, et al. Risk factors and clinical presentation of portal vein thrombosis in patients with liver cirrhosis. J Hepatol 2004;40(5):736-741

15 Wu M, Schuster M, Tadros M. Update on management of portal vein thrombosis and the role of novel anticoagulatns. J Clin Transl Hepatol 2019;7(2):154-164

16 Jüngst C, Krämer J, Schneider G, Lammert F, Zimmer V. Subacute liver failure by pseudocirrhotic metastatic breast cancer infiltration. Ann Hepatol 2013;12(5):834-836

17 Lubner MG, Pickhardt PJ. Multidetector computed tomography for assessment of hepatic fibrosis. Clin Liver Dis (Hoboken) 2018;11(6):156-161

18 Reshamwala PA, Kleiner DE, Heller T. Nodular regenerative hyperplasia: not all nodules are created equal. Hepatology 2006;44(1):7-14

19 Sharma A, Houshyar R, Bhosale P, Choi JI, Gulati R, Lall C. Chemotherapy induced liver abnormalities: an imaging perspective. Clin Mol Hepatol 2014;20(3):317-326 